\title{
Análisis de la influencia de la profundidad de cimentación en la disminución de asentamientos en losas de fundación combinadas con pilotes
}

\section{Analysis of the influence of the foundation depth on the reduction of settlements in piled raft} foundations

Fecha de entrega: 19 de diciembre 2016 Fecha de aceptación: 5 de octubre 2017

\section{Luis O. Ibáñez Mora}

Facultad de Construcciones, Universidad Central Marta Abreu de Las Villas, Carretera de Camajuaní km 5 1/2, Santa Clara, Villa Clara, CP 408000,Cuba, ibanez@uclv.edu.cu

El empleo de losas de fundación combinadas con pilotes constituye una variante de cimentación en aquellos terrenos muy deformables sometidos a grandes cargas. De vital importancia en el proceso de diseño es estimar los asentamientos que se pueden generar y proponer una cantidad de pilotes que garanticen se cumpla con los criterios de capacidad de carga y asentamientos admisibles. Resultados experimentales sugieren que en la medida en que se aumente la profundidad de cimentación, esta comienza a trabajar como un cimiento compensado y disminuyen los asentamientos totales. En este trabajo se muestran los resultados obtenidos con el empleo de programas computacionales profesionales y hojas de cálculo programadas en MathCad, para determinar la curva estimada carga versus asentamiento. Se demuestra la influencia de la profundidad de cimentación en la disminución de los asentamientos en fundaciones flotantes combinadas con pilotes.

Palabras clave: pilotes, fundaciones flotantes, modelación numérica, diseño, asentamiento
The employment of piled raft foundations constitutes a variant offoundation in grounds with very compressible soils and under large loads. Of vital importance in the design process it is the estimation of the settlement and the proposal of the quantity of piles that guarantee the compliance of the criteria of bearing capacity and allowable settlements. Experimental results suggest that when the depth of foundation increases, the foundation begins to work as a compensated foundation diminishing the total settlements. In this work, results of loads versus settlements obtained with the employment of professional computational programs and spreadsheets programmed in MathCad are shown. It is demonstrated the influence of the depth of foundation on the decrease of the settlement in piled raft foundations.

Keywords: piles, compensated foundation, numerical modelling, design, settlement

\section{Introducción}

Las losas de fundación combinadas con pilotes LCP, también conocidas como balsas combinadas con pilotes $\mathrm{BCP}$ o fundación flotante con pilotaje, constituye un sistema constructivo caracterizado por la acción conjunta de ambos elementos: losa y pilotes, con la función de transmitir al terreno las cargas de la superestructura que se ha de cimentar. En las últimas décadas son numerosas las investigaciones y desarrollos conceptuales que han conducido a mejorar el conocimiento del comportamiento conjunto de la losa y pilotes (Cunha, 2000a,b, 2001; Fleming et al.,
2009; O’Neill, 2001; Poulos, 2001a,b; Van Impe y Lungu, 1996), y que hacen de este sistema constructivo una alternativa de cimentación asociada en general a edificios en altura cimentados sobre suelos granulares y en particular sobre suelos arcillosos en estado normalmente consolidados o sobre consolidados. A pesar de que esta variante viene usándose con cierta frecuencia, en la mayoría de los casos su proyecto se apoya en la intuición y experiencia del ingeniero más que en un análisis debidamente fundamentado. El análisis de la interacción entre los tres elementos: losa, pilotes y suelo es de primordial importancia 
para la evaluación de los asentamientos del conjunto, sean uniformes o diferenciales. A su vez, una acertada predicción de los asentamientos es indispensable para verificar que los requerimientos en el estado de servicio se vean cumplidos. Resumidamente, la losa y los pilotes son responsables de transferir las cargas actuantes al terreno, activando la capacidad portante de las distintas capas de este último. Además de esto, la cimentación en LCP tiene efectos positivos adicionales respecto a una losa de cimentación única como:

1. Aumento de la capacidad portante de la cimentación

2. Limitación de la descarga del suelo durante la ejecución de la excavación, debido a que los pilotes, que en esta etapa trabajan a tracción, previenen la liberación de tensiones en el mismo. Evitando los levantamientos excesivos de la base de la excavación.

3. Reducción de las tensiones transmitidas al suelo a través de la losa de cimentación mediante una adecuada elección del número y disposición de los pilotes.

4. Reducción del riesgo de fallas o fisuras en elementos de la superestructura, en particular las fachadas de los edificios.

5. Implementación de un bloque excéntrico que impide el volcamiento en el caso de cargas actuantes excéntricas, mediante un arreglo asimétrico de los pilotes.

En este trabajo se analizará la influencia de la profundidad de cimentación en la disminución de asentamientos en las LCP, principio de trabajo de las cimentaciones compensadas.

\section{Cimentaciones compensadas}

Las cimentaciones totalmente compensadas son aquellas en que el peso de la estructura es igual a la carga del material excavado, en donde se va a construir la cimentación que soportará dicha estructura. Por su parte las cimentaciones parcialmente compensadas pueden ser de dos tipos:
1. Cimentación sub compensada: el peso de la estructura es menor que la carga del material excavado

2. Cimentación sobre compensada: el peso de la estructura es mayor que la carga del material excavado

El empleo de cimentaciones compensadas inicialmente se remonta a la necesidad de garantizar estructuras de cimentación en suelos muy compresibles, donde se garantice el criterio de asentamiento sin la necesidad de acudir a cimentaciones sobre pilotes (Juárez y Rico, 1969; Jiménez et al., 1986). En cimentaciones compensadas total o parcialmente, se busca reducir los incrementos de esfuerzos netos a distintas profundidades, por medio de una excavación (sótano). Para ello, en el cálculo de los asentamientos deberá restarse a los esfuerzos trasmitidos por la estructura, el esfuerzo producto del material excavado. La Figura 1 muestra el efecto de sótano en el alivio de esfuerzos debido a la excavación de profundidad $D$ y suelo de peso unitario $\gamma$ viene dado por $q_{\text {exc }}=D \gamma$, siendo la carga total del edificio $q_{\text {edificio }}$ igual al peso del edificio $P_{\text {edificio }}$ dividido por el ancho y largo del edificio en planta, $q_{\text {edificio }}=P_{\text {edificio }} / B L$. Se tiene entonces que $q_{\text {exc }}$ ya generó asentamientos antes de la construcción y que solo parte de $q_{\text {edificio }}$ es nueva para el suelo y ocasiona asentamientos. Por lo tanto se tiene por lo tanto que $q_{\text {neta }}=q_{\text {edificio }}-q_{\text {exc }}$ y el cálculo del incremento de carga vertical $\Delta \sigma_{z}$ se debe hacer con $q_{\text {neta }}$.

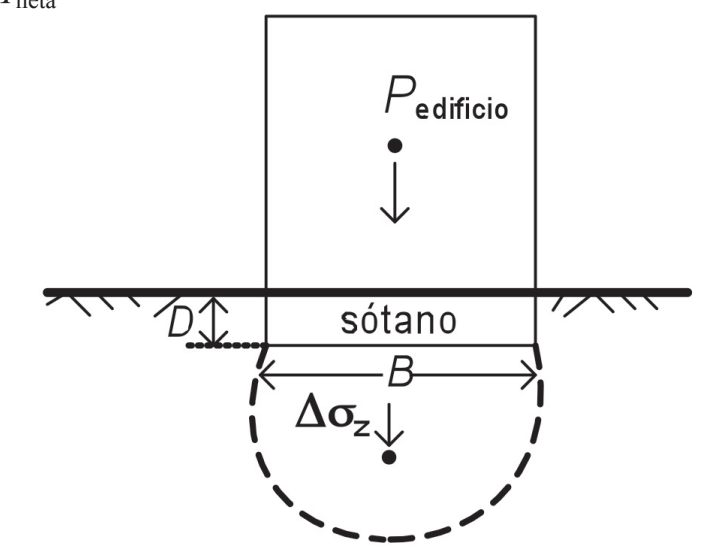

Figura 1: Cimentaciones compensadas

Adicionalmente, deberá trabajarse con los rangos de preconsolidación de las curvas esfuerzo y 
asentamiento, en las áreas donde la remoción de la carga haya producido preconsolidación del material. Debe verificarse que la presión neta tenga un factor de seguridad mínimo de 1.5 contra asentamientos, respecto a la presión de preconsolidación en los suelos influenciados por el cajón de sótano, con el fin de evitar asentamientos excesivos. La remoción de la sobre carga cuando se excava al nivel de la cimentación causa una expansión del fondo de la excavación. Después de aplicar la carga el suelo se recomprime, después de que la carga se incrementa hasta la presión original de la sobrecarga, ocurre la recompresión inmediata. Estos movimientos son insignificantes en caso de cimentaciones poco profundas pero se deben tomar en cuenta en cimentaciones profundas (Tomlinson, 1986).

\section{Cálculo de asentamientos en cimentacio- nes compensadas}

El incremento de edificios de gran altura alrededor del mundo y la necesidad de utilizar el espacio bajo la superficie ha dado como resultado el surgimiento de muchos proyectos con varios niveles de sótanos. Cuando en estas estructuras se utiliza la cimentación LCP, el proceso de construcción y la aplicación o removida de cargas son factores muy importantes en el comportamiento final de la cimentación. El proceso de excavación e instalación de los pilotes también es un factor importante. En edificios con excavaciones superficiales la instalación de los pilotes puede ser ejecutada antes de la excavación (Figura 2). En otros casos cuando debe ser alcanzada una gran profundidad, los pilotes pueden ser colocados luego de la excavación.

Otros de los aspectos a tener en cuenta para determinar los asentamientos en la cimentación son: el proceso de excavación, el tiempo entre el fin de la excavación y la construcción de la balsa, la variación del nivel freático y el tiempo de la construcción. Considerando lo anteriormente dicho (Sales et al., 2009) presentan un método de análisis simplificado. Este método puede ser aplicado en tres etapas como se muestra en la Tabla 1.

a)
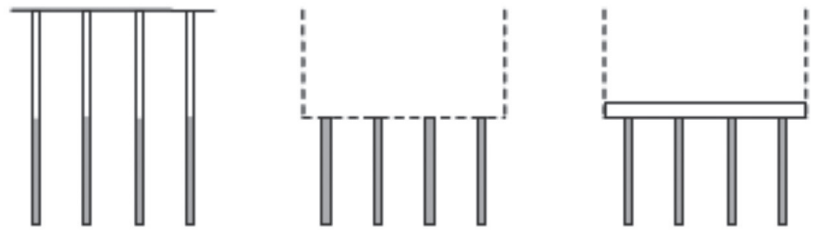

b)
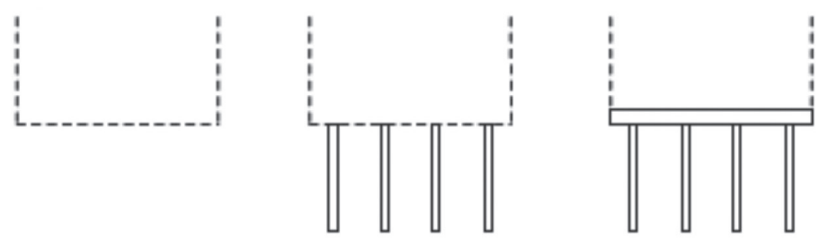

Figura 2: Diferentes formas de construcción de LCP compensadas, a) los pilotes se colocan antes de la excavación y b) los pilotes se colocan después de la excavación (Poulos, 2001a)

\section{Métodos de análisis de balsas combinadas con pilotes}

Varios métodos de análisis para LCP se han desarrollado, entre los mismos se reconocen los métodos de cálculo simplificado, los métodos

Tabla 1: Etapas de análisis para una cimentación en LCP (Sales et al., 2009)

\begin{tabular}{|c|c|c|c|c|c|c|}
\hline Etapas & Período de construcción & $\begin{array}{c}\text { Carga } \\
\text { total } V\end{array}$ & Condiciones del suelo & \multicolumn{2}{|c|}{ Cálculos } \\
\cline { 4 - 6 } & $1 \mathrm{~A}$ & Construcción balsa & $V=R f$ & $\begin{array}{c}\text { Sobre consolidado, no } \\
\text { drenado }\end{array}$ & $S_{0}$ & $P_{0}^{\prime}$ \\
\hline $1 \mathrm{~B}$ & $\begin{array}{c}\text { Comienzo de la } \\
\text { estructura }\end{array}$ & $R f<V \leq E x$ & $\begin{array}{c}\text { Sobre consolidado, no } \\
\text { drenado }\end{array}$ & $S_{0}+\Delta S_{1}$ & $P_{0}^{\prime}+\Delta P_{1}^{\prime}$ & $R_{1}^{\prime}=R_{0}^{\prime}+\Delta R_{1}^{\prime}, R_{1}$ \\
\hline 2 & $\begin{array}{c}\text { Comienza a actuar la } \\
\text { carga de la estructura }\end{array}$ & $E x^{\prime}<V \leq G$ & Estado no drenado & $S_{0}+\Delta S_{2}$ & $P_{0}^{\prime}+\Delta P_{2}^{\prime}$ & $R_{2}^{\prime}=R_{1}^{\prime}+\Delta R_{2}^{\prime}, R_{2}$ \\
\hline 3 & $\begin{array}{c}\text { Periodo después de la } \\
\text { construcción }\end{array}$ & $V=G+P$ & Estado drenado & $S_{0}+\Delta S_{3}$ & $P_{0}^{\prime}+\Delta P_{3}^{\prime}$ & $R_{3}^{\prime}=R_{1}^{\prime}+\Delta R_{3}^{\prime}, R_{3}$ \\
\hline
\end{tabular}

$R f$ : peso de la balsa; $S$ : asentamiento; $P^{\prime}$ : carga que actúa sobre el grupo de pilotes; $R$ : carga resultante de la balsa; $R$ ': carga de la balsa; $E x^{\prime}$ : carga efectiva del suelo excavado; $\Delta P^{\prime}$ : incremento de la carga efectiva del grupo de pilotes; $\Delta R^{\prime}:$ incremento de la carga efectiva en la balsa; $G$ : carga muerta (peso de la estructura), varía con el tiempo; $P$ : carga viva; 1,2 y 3 : etapas de construcción 
computacionales aproximados y los métodos computacionales rigurosos. Entre los métodos simplificados, podemos citar los propuestos por Poulos y Davis (1980), Randolph y Wroth (1979), Randolph (1994), Van Impe y De Clerq (1995) y Burland (1995). En todos ellos se realiza una simplificación en la modelación del suelo, y la forma de aplicación de la carga. A continuación se describe el método de Poulos y Davis (1980), utilizado en este trabajo el desarrollo de las hojas de cálculo en MathCad. Las hipótesis del mismo se basan en considerar que la capacidad de carga última de la cimentación en su conjunto bajo carga vertical toma uno de los siguientes valores:

- La suma de la capacidad de carga de la balsa más la capacidad de carga de todos los pilotes o

- La suma de la capacidad de carga del bloque que conforman los pilotes y la balsa, más la porción restante de la balsa

Es importante destacar que la capacidad de carga del pilote aislado se verá afectado por el efecto de pilotes vecinos, lo que se conoce como eficiencia de grupo y puede variar entre $\xi=0.7$ a 1 . Para estimar el comportamiento de la fundación usando la curva carga versus asentamiento de la cimentación balsa con pilotaje, se aplica las expresiones propuestas por Randolph (1994) y se determina la rigidez de la cimentación LCP como:

$$
K_{p g}=\frac{K_{p}+K_{r}\left(1-\alpha_{c p}\right)}{1-\alpha_{c p}^{2}\left(K_{r} / K_{p}\right)}
$$

Donde $K_{\mathrm{pg}}$ es la rigidez de la cimentación balsa pilote, $K_{\mathrm{p}}$ es la rigidez del pilote, $K_{\mathrm{r}}$ es la rigidez de la balsa y $\alpha_{\text {cp }}$ es un factor de interacción balsa pilote. El valor de $\alpha_{c p}$ según Randolph y Clancy (1994) puede adoptarse igual a 0.8 para grandes grupos de pilotes. La porción de la carga total que toma la losa se expresa como:

$$
X=\frac{K_{r}\left(1-\alpha_{c p}\right)}{K_{p}+K_{r}\left(1-\alpha_{c p}\right)}
$$

Conociendo estos coeficientes se puede confeccionar la curva estimada carga versus asentamiento de la cimentación tal como se muestra en la Figura 3.

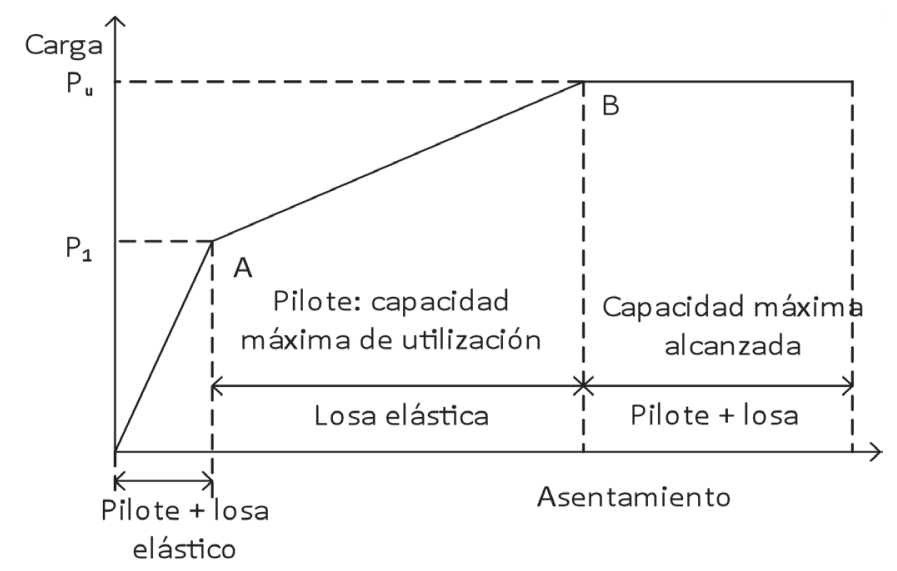

Figura 3: Curva carga versus asentamiento para la cimentación de losa combinada con pilotes

Varios programas de cómputo también han sido desarrollados para el análisis de LCP, entre los que se pueden citar el programa Tief Geotec (2007) y Plaxis (2004). El programa Plaxis fue concebido inicialmente para el análisis de diques construidos sobre las capas de suelo blando que forman el subsuelo de Holanda. Actualmente, las aplicaciones de Plaxis engloban la mayoría de los problemas de ingeniería geotécnica, tanto de mecánica de suelos como de mecánica de rocas. Aunque las aplicaciones del programa en problemas de geotecnia parecen estar totalmente resueltos, en realidad no es así, ya que los programa han sido desarrollado partiendo de la filosofía inicial del estudio del subsuelo sometido a grandes cargas y a grandes excavaciones subterráneas, incluyendo en todos ellos el problema de flujo y ofreciendo la posibilidad del análisis mediante diferentes modelos de comportamiento del terreno.

El ejemplo real a analizar en el trabajo es el propuesto por O'Neill et al. (2001), este consiste en una balsa de cimentación de $5 \mathrm{~m}$ x $5 \mathrm{~m}$ y un espesor de 0.5 $\mathrm{m}$ y con cuatro pilotes de $16 \mathrm{~m}$ de longitud y $0.5 \mathrm{~m}$ de diámetro, espaciados a $3 \mathrm{~m}$. Las condiciones del subsuelo son similares a las de Surfers Paradise en Australia y son resultado de ensayos realizados en el terreno, las propiedades del suelo adoptadas para el análisis se pueden observar en la Figura 4. El suelo de Surfers Paradise tiene una elevada capacidad de carga en la superficie lo que hace favorable el uso de 
cimentaciones en balsa, pero la alta compresibilidad de la turba orgánica puede ser el causante de asentamientos excesivos para los edificios cimentados en esa zona, es por esto la necesidad del uso de cimentaciones en LCP.

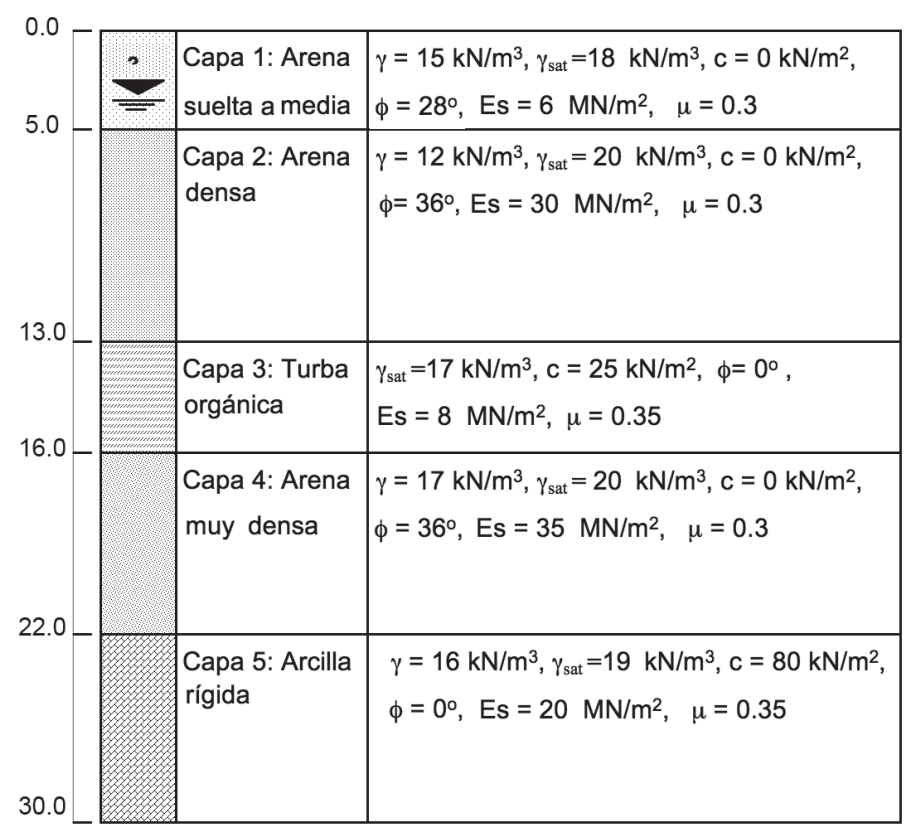

Figura 4: Propiedades del suelo utilizadas en el análisis (O’Neill et al., 2001)

Para la modelación del problema inicialmente se utiliza el programa Plaxis. Para la modelación del suelo y basados en el material que lo compone (arena principalmente) es preferible utilizar el criterio de falla de Mohr-Coulomb por su simplicidad y rapidez para realizar un análisis preliminar. La balsa fue modelada utilizando el comando Floor y los pilotes utilizando Pile con los apropiados parámetros geométricos $\mathrm{y}$ geotécnicos que se pueden ver resumidos en la Tabla 2. La cimentación deberá soportar una carga de servicio de $215 \mathrm{kN} / \mathrm{m}^{2}$.

En la Figura 5 se puede observar el modelo de la cimentación realizado en el programa Plaxis y en la Figura 6 se presenta la curva carga versus asentamiento obtenida como resultado del análisis. En terreno se obtuvo un asentamiento máximo de $0.335 \mathrm{~m}$. Los asentamientos absolutos fueron medidos desde la construcción del edificio en Surfers Paradise en Australia hasta los 10 años de vida de esta edificación, con la utilización de las mediciones topográficas realizadas (O’Neill et al., 2001).
Tabla 2: Parámetros utilizados en el análisis

\begin{tabular}{|l|c|c|}
\hline Parámetro & Balsa & Pilote \\
\hline $\begin{array}{l}\text { Módulo general de deformación } \\
E, \mathrm{MPa}\end{array}$ & 34000 & 23500 \\
\hline Razón de Poisson & 0.2 & 0.2 \\
\hline Espesor/diámetro, m & 2.5 & 0.9 \\
\hline Cantidad & 1 & 4 \\
\hline
\end{tabular}

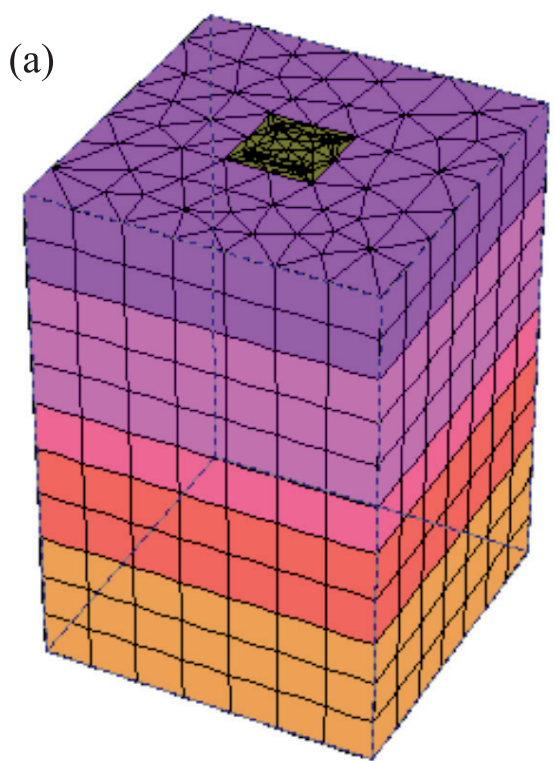

(b)

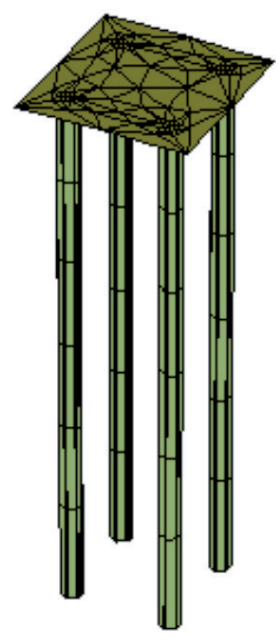

Figura 5: a) Modelación de las capas de suelo y b) de la cimentación en LCP

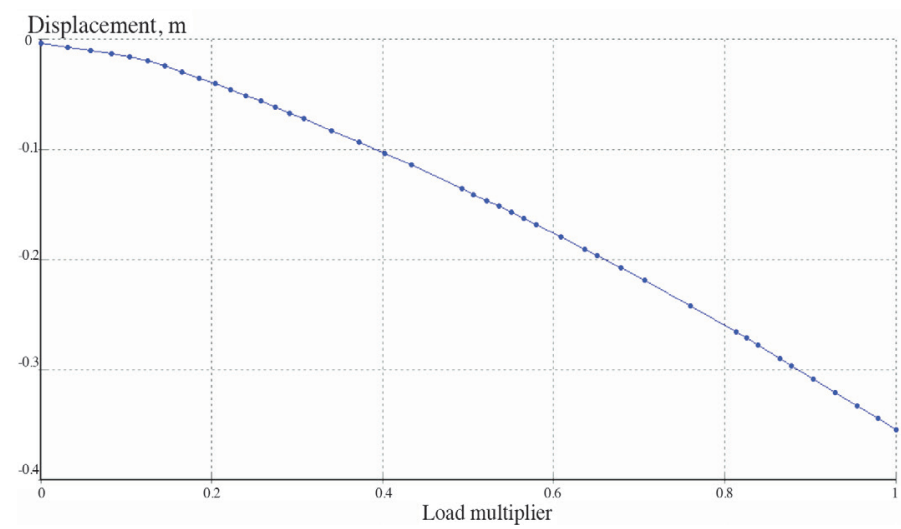

Figura 6: Curva carga versus asentamiento U obtenida para una profundidad de cimentación de $0 \mathrm{~m}$

Los valores de asentamiento estimados para esta cimentación con el uso de las hojas de cálculo programadas en MathCad (2004) y Tief Geotec fueron algo mayores, pero casi similares y muy cercanos a los valores medidos en terreno como se puede observar en la Tabla 3. 
Tabla 3: Asentamiento obtenido con el uso de diferentes programas computacionales

\begin{tabular}{|l|c|}
\hline Programa utilizado & Asentamiento, $\mathrm{cm}$ \\
\hline Plaxis & 35.5 \\
\hline Tief Geotec & 37.9 \\
\hline MathCad & 36.6 \\
\hline Valores medidos en el terreno & 33.5 \\
\hline
\end{tabular}

Estos resultados demuestran la validez de los modelos utilizados con los software Plaxis y Tief Geotec, además de las potencialidades del empleo de hojas de cálculo como ayuda en el análisis y solución de problemas ingenieriles complejos.

\section{Efecto de la profundidad de cimentación en la modelación de LCP}

Con el fin de analizar la influencia de la profundidad de cimentación en el comportamiento de LCP se realizaron tres modelos en los programas Plaxis, Tief Geotec y las hojas de cálculo en MathCad para profundidades de cimentación de 0,3 y $6 \mathrm{~m}$. Para una profundidad de cimentación diferente de 0 las paredes de la excavación fueron modeladas utilizando el comando Wall en el programa Plaxis. En la Figura 7 se observa el modelo de la cimentación en LCP para una profundidad de cimentación de 3 y $6 \mathrm{~m}$.

Como se observa en la Figura 8 el aumento de la profundidad de cimentación en cimentaciones en LCP trae consigo una disminución considerable en los asentamientos del sistema. También se puede observar en la Tabla 5 y en la Figura 9 que los resultados obtenidos con el uso de los programas Plaxis, Tief Geotec y las hojas de cálculo son similares, demostrando una vez más que son un medio seguro y sencillo para realizar el análisis de cimentaciones en LCP.

Tabla 5: Resultados obtenidos para profundidades de cimentación de 0,3 y $6 \mathrm{~m}$

\begin{tabular}{|c|c|c|c|}
\hline \multirow{2}{*}{$\begin{array}{c}\text { Profundidad de } \\
\text { cimentación } D_{\mathrm{f}}, \mathrm{m}\end{array}$} & \multicolumn{3}{|c|}{ Asentamiento, cm } \\
\cline { 2 - 4 } & Plaxis & Tief Geotec & MathCad \\
\hline 0 & 35.50 & 37.94 & 36.62 \\
\hline 3 & 14.80 & 17.20 & 15.68 \\
\hline 6 & 11.40 & 9.74 & 9.02 \\
\hline
\end{tabular}

(a)
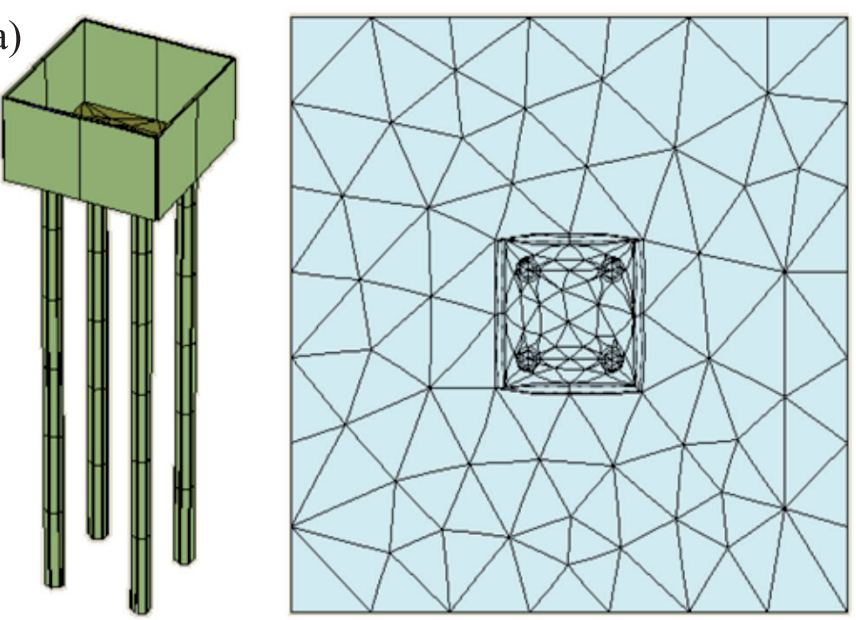

(b)

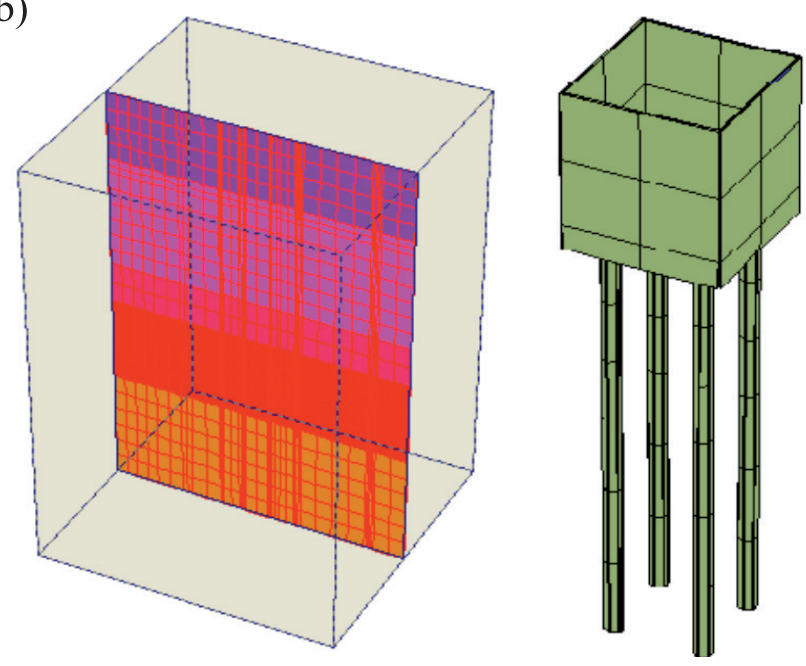

Figura 7: Modelación de la cimentación en LCP para profundidades de a) 3 y b) $6 \mathrm{~m}$

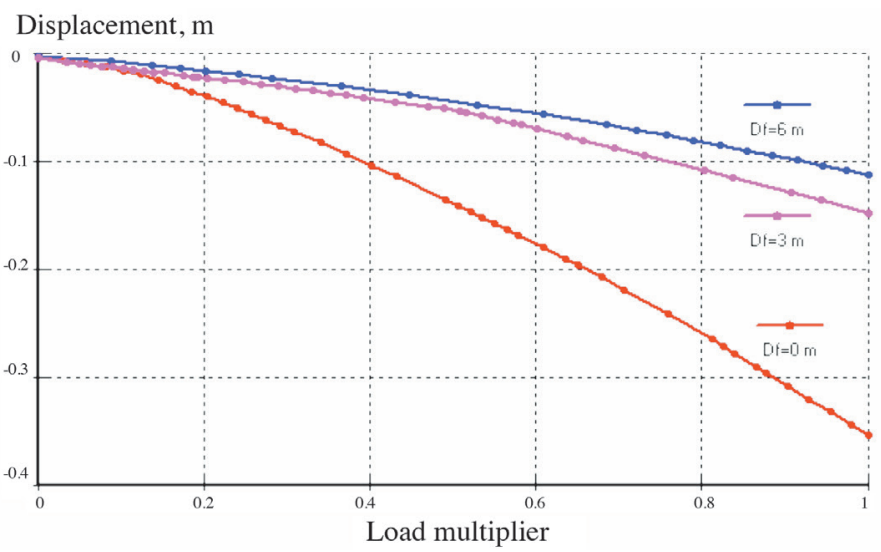

Figura 8: Curvas carga versus asentamiento para profundidades de cimentación $D_{\mathrm{f}}$ de 0,3 y $6 \mathrm{~m}$ 


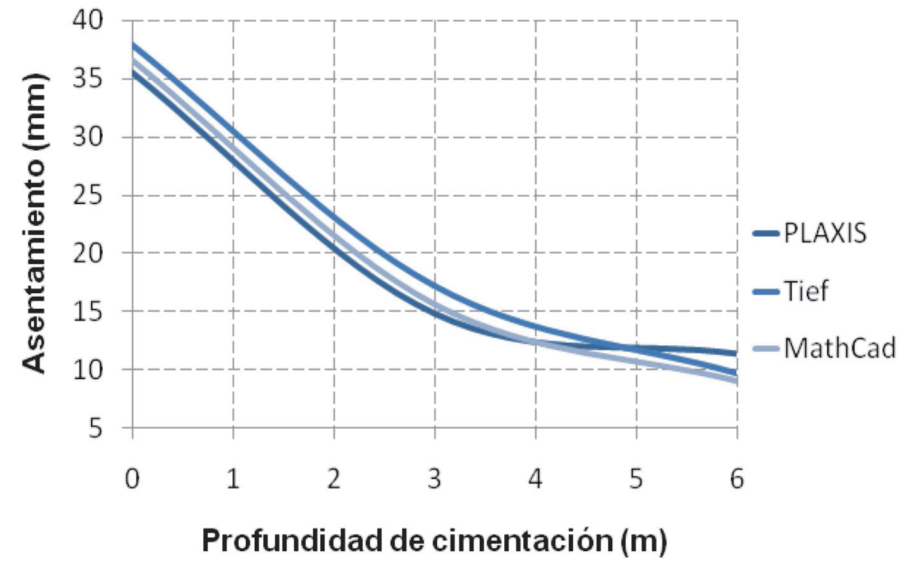

Figura 9: Resultados obtenidos para profundidades de cimentación de 0,3 y $6 \mathrm{~m}$

Como se observa en la Figura 9, el aumento de la profundidad de cimentación, provoca una disminución en los asentamientos, lo que se explica porque el esfuerzo que transmite la estructura se ve contrarrestado por el material excavado, principio de trabajo de las cimentaciones compensadas y en correspondencia con lo propuesto por Sales et al. (2009).

\section{Conclusiones}

Como conclusiones de este trabajo se pueden señalar que se demuestra la validez del empleo de cimentaciones flotantes combinadas con pilotes como una cimentación adecuada para soportar grandes cargas y obtener menores asentamientos. Finalmente, el empleo de modelos simplificados para el análisis de cimentaciones flotantes combinadas con pilotes permiten obtener resultados satisfactorios, próximos a los medidos en terreno y similares a los obtenidos con programas computacionales complejos.

\section{Agradecimientos}

El autor agradece a la Universidad de Brasilia, en especial al Programa de Post Graduado en Geotecnia y de manera destacada al Profesor Renato P. Cunha. A CAPES/Brasil por el apoyo financiero para la realización de este trabajo y estancia en la Universidad de Brasilia.

\section{Referencias}

Burland, J.B. (1995). Piles as settlement reducers. 19th Italian Congress on Soil Mechanics, Pavia, Italy, 21-34

Cunha, R.P., Poulos, H.G. and Small, J.C. (2001). Investigation of design alternatives for a piled raft case history. Journal of Geotechnical and Geoenvironmental Engineering 127(8), 635-641

Cunha, R.P., Small, J.C. and Poulos, H.G. (2000a). Parametric analysis of a piled raft case history in Uppsala, Sweden. $4^{\circ}$ Seminário de Engenharia de Fundações Especiais e Geotecnia, São Paulo, vol. 2, 381-390

Cunha, R.P., Small, J.C. and Poulos, H.G. (2000b). Class $\mathrm{C}$ analysis of a pile raft case history in Gothenburg, Sweden. Conference on Developments in Geotechnical Engineering, Thailand, vol. 1, 271-280

Fleming, K., Weltman, A., Randolph, M. and Elson, K. (2009). Piling engineering. Taylor \& Francis

Jiménez Salas, J.A., de Justo Alpañes, J.L. y Serrano González, A.A. (1986). Geotecnia y cimientos. Editorial Rueda. Madrid, tomos II y III

Juárez Badillo, E. y Rico Rodríguez, A. (1969). Mecánica de suelos. Edición Revolucionaria, La Habana, tomos I y II MathCad (2004). User guide MathCad version 14. http:// www.mathsoft.com

O’Neill, M.W., Caputo, V., De Cock, F., Hartikainen, J. and Mets, M. (2001). Case histories of pile-supported rafts. Report for ISSMFE TC18, University of Houston, Texas

Plaxis (2004). Plaxis 3D Foundations. Tutorial manual version 1.6.

Poulos, H.G. (2001a). Methods of analysis of piled raft foundations. Report Prepared on TC18 report on Piled Foundations, ISSMGE

Poulos, H.G. (2001b). Piled raft foundations: design and applications. Géotechnique 51(2), 95-113

Poulos, H.G. and Davis, E.H. (1980.). Pile foundation analysis and design. John Wiley and Sons 
Randolph, M.F. (1994). Design methods for pile groups and piled rafts. 13th International Conference on Soil Mechanics and Foundation Engineering, New Delhi, vol. $5,61-82$

Randolph, M. and Clancy, P. (1994). Design and performance of a piled raft foundation. Conference on Vertical and Horizontal Deformations of Foundations and Embankments. Settlement'94, ASCE GSP, 314-324

Randolph, M.F. and Wroth C.P. (1979). An analysis of the vertical deformation of pile groups. Géotechnique 29(4), 423-439

Sales, M.M., Small, J.C. and Poulos, H.G. (2009). Compensated piled rafts in clayey soils: behaviour, measurements, and predictions. Canadian Geotechnical Journal 47(3), 327-345
Tief Geotec (2007). Geotec Office 9.2 SP 1, tutorial manual.

Tomlinson, M.J. (1986). Foundation design and construction. Longman Scientific and Technical, Harlow, 5 th edn.

Van Impe, W.F. and De Clerq, Y. (1994). A piled raft interaction model. Fifth International Conference on Piling and Deep Foundations, Bruge, 1.3.1-1.3.10

Van Impe, W.F. and Lungu, I. (1996). Technical report on settlement prediction methods for piled raft foundations. Ghent University, Belgium 